\title{
La adquisición del parámetro de ascenso verbal en el alemán como tercera lengua
}

\author{
María MarTínEZ Adrí́n \\ Universidad del País Vasco
}

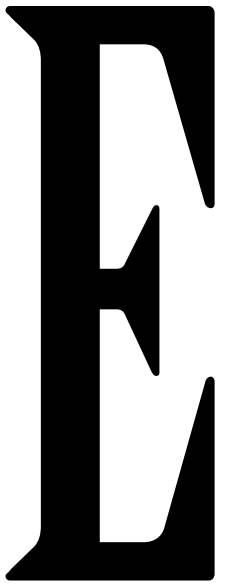

n este trabajo ${ }^{1}$ abordamos el tema de la posible transferencia de una segunda lengua (L2) a una tercera lengua (L3), concretamente de inglés como L2 a alemán como L3. Nos centramos en las dificultades a las que se enfrentan los estudiantes en lo que respecta a la adquisición del orden de palabras de la oración alemana e investigamos si el inglés como L2 puede tener influencia en el aprendizaje de los distintos órdenes de la oración alemana puesto que estas lenguas guardan semejanzas a nivel léxico, fonológico o gramatical y pertenecen a la misma familia. También partimos del hecho de que el estudiante de lenguas extranjeras puede relacionar una lengua con otra y percibir la distancia y grado de similitud entre una lengua origen y otra meta (Kellerman 1977, 1986, 1987).

Como marco teórico para el análisis de las distintas estructuras estudiadas hemos elegido el Programa Minimalista (PM) (Chomsky 1994, 1995) y, concretamente, la propuesta que realiza Zwart (1997ab) para lenguas germánicas como el holandés o el alemán, entre otras. Con respecto al parámetro de ascenso verbal y a las lenguas implicadas en este trabajo, podemos caracterizar dichas lenguas en función de la fortaleza de los rasgos de concordancia. Así, lenguas como el euskera, el castellano y el alemán son lenguas de rasgos fuertes, a diferencia del inglés, cuyos rasgos son débiles. Por ello, formulamos la hipótesis de una posible transferencia del rasgo [+débil] del inglés al verbo alemán.

En nuestro entorno se han realizado investigaciones sobre adquisición de L3, concretamente de aprendizaje de inglés como L3 (Cenoz 2001; García Mayo 2003). Sin embargo, no se han llevado a cabo estudios sobre adquisición de otra lengua extranjera después del inglés, salvo nuestros estudios pilotos sobre la adquisición de los auxiliares de perfecto y el orden de palabras del alemán (Martínez Adrián 2001, 2002) que nos han ofrecido las pautas para el diseño de la presente investigación. Aunque los trabajos realizados fuera de nuestras fronteras como Bouvy (2000), Gibson et al. (2001) o Vogel (1992), entre otros, nos proporcionan evidencia de influencia del inglés como L2 a distintos niveles, no hemos encontrado trabajos sobre transferencia del inglés como L2 en el plano sintáctico y enmarcados dentro del PM. Por otro lado, gran parte de estos trabajos se han realizado con una muestra muy limitada. Nuestra investigación ofrece un aspecto novedoso: no sólo contamos con una muestra amplia sino con variedad de pruebas administradas.

Con objeto de investigar la hipótesis de la transferencia de la L2, hemos obtenido datos de cuatro grupos que aprenden ale- mán como L3 y de tres grupos de control cuya L2 es el alemán. Las pruebas administradas a estos grupos se complementan puesto que unas abarcan el ámbito de la producción y otras el de la comprensión.

\section{La adquisición de segundas lenguas y el fenómeno de la transferencia}

La influencia de la primera lengua (L1) en el aprendizaje de la L2, a la que de aquí en adelante nos referiremos como transferencia, ha dado lugar a un gran número de estudios que se han enmarcado dentro de teorías conductistas y cognitivistas. Así, desde un punto de vista conductista, la influencia de la L1 se ve como un impedimento para aprender la L2 si esas dos lenguas difieren de manera relevante (Fries 1945; Lado 1957). Desde los enfoques cognitivistas (Faerch y Kasper 1986; Kellerman 1986; Kohn 1986), la transferencia se ve como proceso cognitivo en el que intervienen diferentes factores tanto estructurales (nivel de lengua, distancia lingüística, psicotipología) como no estructurales (lugar de aprendizaje, metodología).

Otra parte de los estudios sobre transferencia se centra en la influencia de la L2 en la L3, aunque hay que apuntar el hecho de que éstos son más escasos. A lo largo de los últimos cuarenta años aproximadamente, se han ofrecido toda una serie de explicaciones para el fenómeno de la transferencia lingüística. Algunos trabajos utilizan (I) el factor de la psicotipología y la distancia lingüística para explicar dicha transferencia (Cenoz 1998; Möhle 1989; Vildomec 1963, entre otros), (II) otros trabajos se centran en aspectos sociales o psicológicos como Meisel (1983), (III) las investigaciones como las de White (1987) tienen en cuenta el concepto de la marcación y (IV) otros estudios se basan en el proceso de aprendizaje con sus respectivos estadios (Corder 1979; Fathman 1989; Ringbom 1983, entre otros). Finalmente, autores como Cenoz (2001) consideran varios factores determinantes como distancia lingüística y psicotipología, nivel de proficiencia, contexto, efecto de la lengua extranjera, edad y recencia.

Y finalmente, nos centramos en los trabajos sobre influencia del inglés como L2 en la adquisición del alemán como L3, puesto que éstas son las lenguas extranjeras implicadas en nuestro estudio. Así, unos se centran en la transferencia a nivel léxico como Ecke y Hall (1998), quienes analizan un corpus de errores léxicos pertenecientes a estudiantes cuya L1 es el castellano, que han estudiado inglés como L2 y se encuentran estudiando el alemán en el momento de realizar la investigación. Estos aprendices muestran una influencia interlingüística mayor del inglés que del castellano. Otros trabajos se centran en la transferencia a nivel gramatical como Welge (1987), Vogel (1992) o Neuner (1996). 
Y por último, trabajos como los de Hakansson et al. (2002) o nuestros estudios previos (Martínez Adrián 2002, 2003) concluyen que no existe influencia del inglés como L2.

\section{La adquisición del orden de palabras}

El estudio de la adquisición de segundas lenguas se ha realizado desde distintas perspectivas. Desde el enfoque chomskiano o más concretamente desde la teoría de Principios y Parámetros, se ha abordado el tema de las diferencias existentes entre la adquisición de una L1 y la adquisición de una L2, o dicho de otra ma-

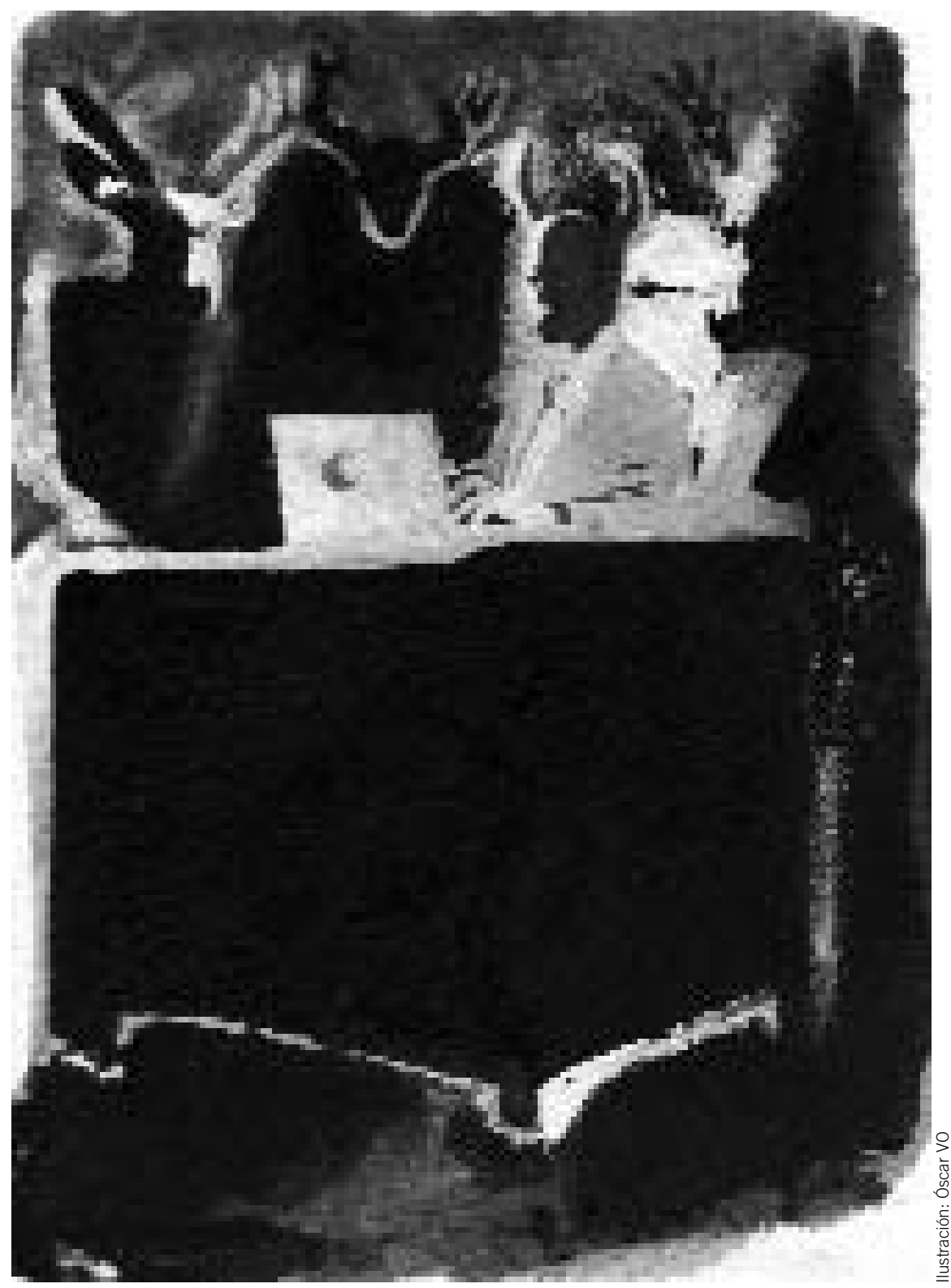

Entre los estudios sobre adquisición del orden de palabras en la L2 que se enmarcan dentro de la temática "acceso a la GU», encontramos los trabajos de Eubank (1993/94), Schwartz y Sprouse $(1994,1996)$, Vainikka y Young-Scholten $(1994,1996)$ y Platzack (1996). Estos autores comparten la idea de la refijación de los parámetros y algunos de ellos la existencia de transferencia en los primeros estadios de aprendizaje. Así, para Schwartz y Sprouse (1994, 1996), existe transferencia absoluta de la L1 y acceso pleno a la GU. Por el contrario, Vainikka y Young-Scholten $(1994,1996)$ defienden la idea de una transferencia parcial, únicamente de proyecciones léxicas. Finalmente, Eubank (1993/94) propone la transferencia de proyecciones léxicas y funcionales de la L1. Sin embargo, sostiene que el valor de los rasgos no se transfiere ya que no se encuentra especificado. En cuanto al movimiento del verbo, Schwartz y Sprouse $(1994,1996)$ proponen que no existe en los primeros estadios pero sí se encuentra movimiento entre los aprendices más expertos. Para Vainikka y YoungScholten (1994, 1996), no existe movimiento del verbo en los primeros estadios y apuestan por la opcionalidad del movimiento en los estadios intermedios. Eubank (1993/94) sugiere que el movimiento es opcional también en los estadios tempranos de aprendizaje puesto que los rasgos son inertes. Pero las tres propuestas coinciden en que el movimiento es obligatorio en los estadios avanzados.

Entre los trabajos que se enmarcan dentro de la temática de «no acceso a la GU», destacamos los de Clahsen (1988) y Meisel (1997) para los que el componente morfológico es independiente de la sintaxis en la adquisición de la L2, a diferencia de la L1, donde se procesan de forma conjunta. Esta idea es retomada en los trabajos de Tsimpli (1997), Hawkins y Chan (1997), Beck (1998ab) o Lardiere (2000). Así por ejemplo, en los estudios de Tsimpli (1997) y Beck (1998a) apreciamos la existencia de movimientos opcionales del verbo como consecuencia de la disociación entre la morfología y la sintaxis. Según la propuesta de Beck (1998ab), todos los aprendices de L2 presentan movimientos opcionales del verbo en todos los estadios de aprendizaje debido a una deficiencia permanente a nivel del valor de fuerza de los

nera, se ha formulado la pregunta de si los principios y parámetros que constituyen la Gramática Universal (GU) siguen operativos en la construcción de las gramáticas en la L2. Es aquí donde surge la discusión en torno a la accesibilidad a la GU. Una de las posturas es la de acceso pleno a la GU y otra la de acceso parcial o nulo a esta dotación genética. rasgos bajo los núcleos funcionales. Sin embargo, para Lardiere (2000), Prévost y White (2000) y Sorace (2000), la opcionalidad de los movimientos no se debe a una deficiencia temporal o permanente, sino a un problema de materialización de los rasgos abstractos. Si los rasgos fuesen inertes o no se encontrasen especificados, no podría haber movimientos opcionales del verbo, es 
decir, el verbo no se movería nunca, ya que el rasgo fuerte es el que motiva el movimiento.

Finalmente, las secuencias de aprendizaje obtenidas a partir del proyecto ZISA (Clahsen, Meisel y Pienemann 1983) (Zweitsprachenerwerb italienischer und spanischer Arbeiter «La adquisición del alemán como L2 de trabajadores italianos y españoles»), llevado a cabo a finales de los años 70, resultan relevantes para cualquier trabajo de adquisición del orden de palabras del alemán. Estos trabajos mostraban datos de inmigrantes españoles e italianos en Alemania y se comparaban con los de la adquisición del alemán como L1. Los investigadores del proyecto llegaron a la conclusión de que una vez superada la primera etapa en la cual el aprendiz producía palabras y fórmulas aisladas, se observaba una secuencia de desarrollo en cinco etapas: SVO < ADV ("Heute ich gehe...") < SEP ("Ich möchte heute ...gehen") < INV "Heute möchte ich...gehen”) < V-FINAL (“.., weil ich... gehen möchte”).

\section{Hipótesis}

Basándonos en los planteamientos del PM y en los estudios de adquisición del orden de palabras en la L2, a los que nos hemos referido en la sección previa, formulamos una serie de hipótesis. A partir de estos estudios previos podríamos establecer tres tendencias que podríamos llegar a observar en nuestros datos: 1. transferencia del inglés como L2; 2 . opcionalidad de los movimientos; 3. refijación del parámetro de ascenso verbal

Por un lado, trataremos de investigar si se produce transferencia de los rasgos de la L2 a la L3. La validez de esta hipótesis la vamos a poner a prueba a través de tres preguntas de investigación:

I. En las oraciones simples tipo SVO en las que se incluyen adverbios, ¿colocan los aprendices de alemán como L3 el adverbio entre el sujeto y el verbo como en la L2, como podemos observar en (1)?

(1) a. *Peter oft nimmt das Auto

Peter a menudo coge el coche

b. Peter nimmt oft das Auto

Peter coge a menudo el coche

«Peter coge el coche a menudo»

L2: «Peter often drives the car»

II. En las oraciones simples tipo SVO con tiempos compuestos como en (2), ¿colocan los aprendices de alemán el adverbio entre el auxiliar y el participio como en la L2?

(2) a. "Inge hat immer geliebt Anton Inge ha siempre amado a Anton

b. Inge hat immer Anton geliebt Inge ha siempre a Anton amado «Inge siempre ha amado a Anton»

L2: «Inge has always loved Anton»

III. En las secuencias VSO, es decir, en las estructuras en las que hay que invertir el sujeto porque existe un elemento en primera posición, ¿se produce la no inversión del sujeto, ilustrada en (3a), como ocurre en la L2?

(3) a. *Nächste Woche Peter isst zu Hause Próxima semana Peter come en casa

b. Nächste Woche isst Peter zu Hause Próxima semana come Peter en casa «La próxima semana come Peter en casa»

\section{L2: «Next week, Peter will eat at home»}

En el caso de que no se produzca transferencia, intentaremos observar si los aprendices de alemán como L3 presentan movimientos opcionales del verbo. Finalmente, si estos dos aspectos no se aprecian en la interlengua de estos aprendices, es decir, si no presentan errores en lo que al orden de palabras se refiere, esto implicaría que han adquirido las estructuras en las que nos hemos centrado en este trabajo.

\section{Metodología}

Con los objetivos e hipótesis planteados, hemos decidido diseñar una investigación de corte trasversal como la que a continuación pasamos a describir.

Este estudio se ha realizado dentro de un marco institucional y por lo tanto, de instrucción formal en el aula. Los datos provienen de siete grupos pertenecientes a tres centros diferentes de Educación Secundaria Obligatoria (E.S.O.) y Educación Primaria (E. P.) de la Comunidad Autónoma del País Vasco.

\section{Participantes}

Los participantes en esta investigación han sido un total de 98 alumnos divididos en siete grupos, cuatro grupos experimentales y tres de control. Las Tablas 1 y 2 resumen la información relativa a cada grupo:

Tabla 1. Grupos experimentales

\begin{tabular}{|c|c|c|c|}
\hline \multicolumn{4}{|l|}{ GRUPO I } \\
\hline $\begin{array}{l}\text { Modelo } A, L 2=\text { inglés } \\
\text { y } L 3=\text { alemán }\end{array}$ & Edad & $\begin{array}{l}\text { Años/Horas semanales } \\
\text { de exposición al inglés }\end{array}$ & $\begin{array}{l}\text { Años/Horas semanales } \\
\text { de exposición al alemán }\end{array}$ \\
\hline $4^{0}$ E.S.O. $(n=12)$ & $15-16$ años & 9 años $/ 3$ horas & 4 años/2 horas \\
\hline \multicolumn{4}{|l|}{ GRUPO II } \\
\hline $\begin{array}{l}\text { Modelo } A, L 2=\text { inglés } \\
\text { y } L 3=\text { alemán }\end{array}$ & Edad & $\begin{array}{l}\text { Años/Horas semanales } \\
\text { de exposición al inglés }\end{array}$ & $\begin{array}{l}\text { Años/Horas semanales } \\
\text { de exposición al alemán }\end{array}$ \\
\hline $3^{0}$ E.S.O. $(n=12)$ & 14-15 años & 11 años/3 horas & 3 años $/ 2$ horas \\
\hline \multicolumn{4}{|l|}{ GRUPO III } \\
\hline $\begin{array}{l}\text { Cursos con Modelo } \mathrm{D} \text {, } \\
\mathrm{L} 2=\text { =inglés y } \mathrm{L} 3=\text { alemán }\end{array}$ & Edad & $\begin{array}{l}\text { Años/Horas semanales } \\
\text { de exposición al inglés }\end{array}$ & $\begin{array}{l}\text { Años/Horas semanales } \\
\text { de exposición al alemán }\end{array}$ \\
\hline $4^{0}$ E.S.O. $(n=20)$ & $15-16$ años & 9 años $/ 3$ horas & 4 años/2 horas \\
\hline \multicolumn{4}{|l|}{ GRUPO IV } \\
\hline $\begin{array}{l}\text { Cursos con Modelo } \mathrm{D} \text {, } \\
\mathrm{L} 2=\text { inglés y } \mathrm{L} 3=\text { alemán }\end{array}$ & Edad & $\begin{array}{l}\text { Años/Horas semanales } \\
\text { de exposición al inglés }\end{array}$ & $\begin{array}{l}\text { Años/Horas semanales } \\
\text { de exposición al alemán }\end{array}$ \\
\hline $3^{0}$ E.S.O. $(n=14)$ & 14-15 años & 8 años $/ 3$ horas & 3 años/2 horas \\
\hline \multicolumn{4}{|c|}{ L1s de los sujetos de los Grupos III y IV } \\
\hline $\mathrm{L} 1$ & Euskera & Castellano & Euskera y castellano \\
\hline GR. III $(n=20)$ & 5 & & 3 \\
\hline GR. IV $(n=14)$ & 4 & 10 & 0 \\
\hline
\end{tabular}




\section{Tabla 2. Grupos de control}

\begin{tabular}{|c|c|c|c|}
\hline \multicolumn{4}{|l|}{ GRUPO V } \\
\hline $\begin{array}{l}\text { Cursos con } L 1=\text { castellano } \\
\text { y } L 2=\text { alemán }\end{array}$ & Edad & $\begin{array}{l}\text { Años/Horas semanales } \\
\text { de exposición al alemán }\end{array}$ & $\begin{array}{l}\text { Horas semanales } \\
\text { de exposición al castellano }\end{array}$ \\
\hline $4^{0}$ E.S.O. $(n=15)$ & 15-16 años & 11 años/23 horas & 4 horas \\
\hline \multicolumn{4}{|l|}{ GRUPO VI } \\
\hline $\begin{array}{l}\text { Cursos con } L 1=\text { castellano } \\
\text { y } L 2=\text { alemán }\end{array}$ & Edad & $\begin{array}{l}\text { Años/Horas semanales } \\
\text { de exposición al alemán }\end{array}$ & $\begin{array}{l}\text { Horas semanales } \\
\text { de exposición al castellano }\end{array}$ \\
\hline $3^{0}$ E.S.O. $(n=12)$ & 14-15 años & 10 años/23 horas & 4 horas \\
\hline \multicolumn{4}{|l|}{ GRUPO VII } \\
\hline $\begin{array}{l}\text { Cursos con } L 1=\text { castellano } \\
\text { y } L 2=\text { alemán }\end{array}$ & Edad & $\begin{array}{l}\text { Años/Horas semanales } \\
\text { de exposición al alemán }\end{array}$ & $\begin{array}{l}\text { Horas semanales } \\
\text { de exposición al castellano }\end{array}$ \\
\hline $4^{0}$ Primaria $(n=13)$ & 9-10 años & 6 años/18 horas & 7 horas \\
\hline
\end{tabular}

\section{Pruebas administradas}

A todos los grupos les hemos administrado pruebas de nivel de alemán (gramática y vocabulario) con el fin de asegurarnos de una competencia adecuada en dicha lengua. Asimismo, a los grupos con alemán como L3, también les hemos administrado una prueba de nivel de inglés (gramática, vocabulario, producción escrita y comprensión escrita) con el fin de comprobar su grado de competencia en esa lengua.

También hemos diseñado cuestionarios en los que se incluye una sección de preguntas personales, una serie de preguntas de orden académico y una sección de preguntas sobre conocimiento lingüístico previo.
En la Tabla 3 se resumen las pruebas utilizadas en este trabajo. Para el estudio de la adquisición del orden de palabras, hemos utilizado dos tipos de pruebas que se complementan puesto que unas abordan el ámbito de la producción, como la redacción y las respectivas traducciones, y otras abordan el ámbito de la comprensión, como la prueba de juicios de gramaticalidad. Actualmente, en la investigación de adquisición de segundas lenguas, la tendencia es utilizar el mayor número de pruebas posibles para triangular datos. Algunas de estas pruebas han sido utilizadas por otros autores como Beck (1998a), Grümpel (2000), Robertson y Sorace (1999) y Tsimpli (1997), entre otros.

Tabla 3. Pruebas utilizadas

Orden de palabras: SVO, VSO, SOV y oraciones coordinadas:

1. Redacción con el título Was hast du heute gemacht? «QQué has hecho hoy?»

2. Juicios de gramaticalidad

3. Prueba de traducción del inglés al alemán de oraciones SVO, VSO, SOV y oraciones coordinadas

4. Prueba de traducción del castellano al alemán de oraciones tipo SVO, VSO, SOV y oraciones coordinadas

5. Prueba de traducción del euskera al alemán de oraciones tipo SVO, VSO, SOV y oraciones coordinadas

6. Introspección

\section{Procedimiento}

Como se puede deducir de la variedad de grupos, nuestro objetivo ha sido por un lado comparar los grupos que aprenden alemán como L3 con los grupos que aprenden alemán como L2 pa-

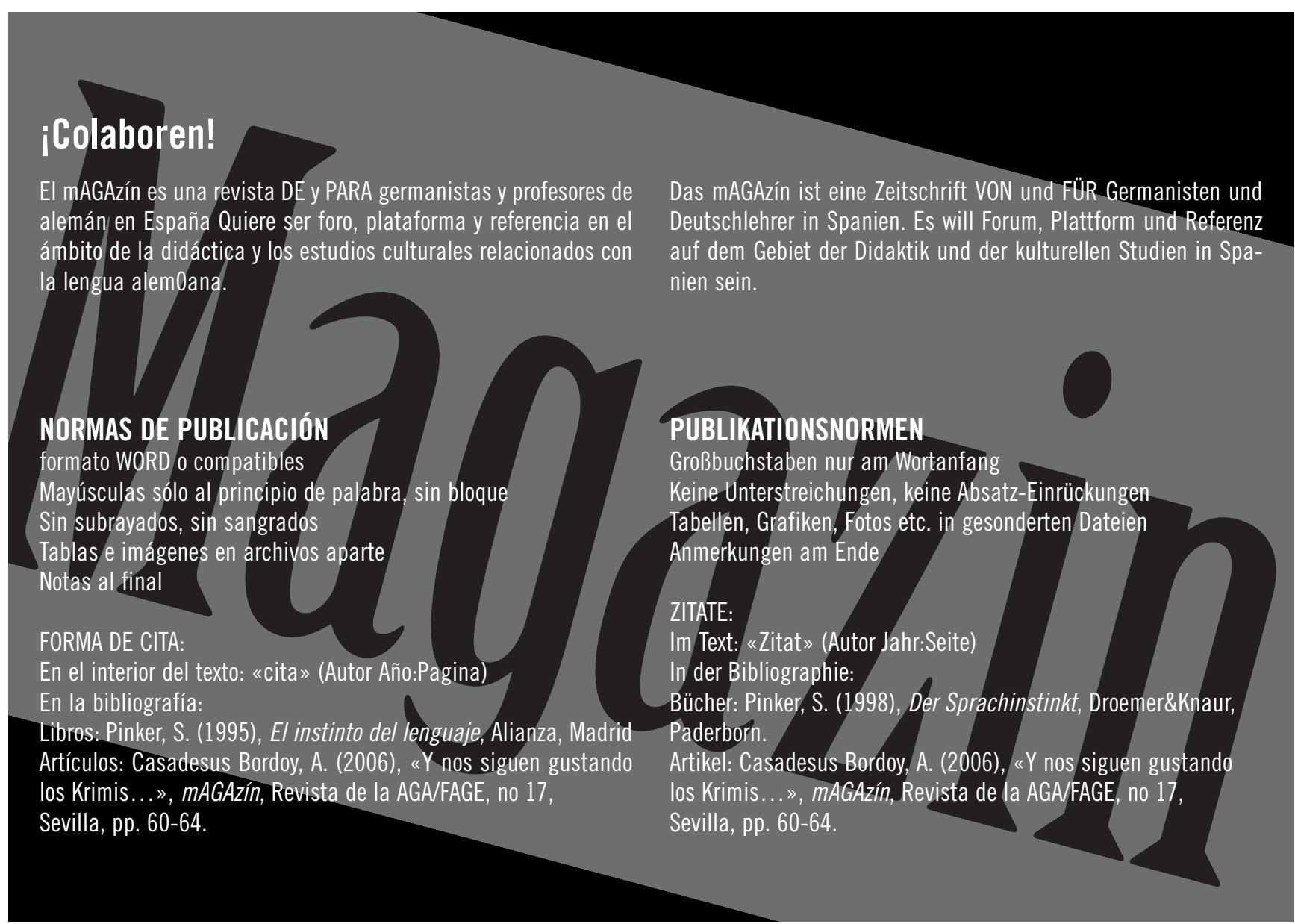


ra saber si el inglés puede tener influencia. A su vez, hemos querido saber si podía existir influencia de la L1 y por eso hemos decidido comparar grupos de Modelo A (cuya lengua de instrucción es el castellano) con grupos de Modelo D (cuya lengua de instrucción es el euskera).

En cuanto al hecho de haber incluido tres grupos de control que aprenden alemán como L2, nuestro fin ha sido comprobar si en estadios anteriores de aprendizaje a los de los grupos V y VI, aparecen errores semejantes a los de los grupos con alemán como L3.

\section{Resultados}

Como ya hemos señalado anteriormente, hemos formulado la hipótesis de una posible transferencia del valor de fuerza de los rasgos del inglés bajo los núcleos funcionales CONC y T. La validez de esta hipótesis la hemos puesto a prueba a través de tres preguntas de investigación. En lo que concierne a la primera pregunta, es decir, si en las oraciones simples en las que se incluyen adverbios, se coloca el adverbio entre el sujeto y el verbo como en la L2, en base a los datos analizados en las distintas pruebas administradas, no podemos concluir que exista influencia de la L2 a la hora de construir oraciones simples en las que se incluyen adverbios por la triple evidencia encontrada, a saber:

(i) hemos comprobado que la secuencia [ ${ }^{*} \mathrm{~S}$ adv VO] no se aprecia de manera generalizada entre los estudiantes de alemán

(ii) en la prueba de traducción de inglés a alemán observamos la colocación del adverbio bien al principio bien al final de la oración, al igual que ocurre tanto en la prueba de traducción euskera-alemán como en la de castellano-alemán (4):

(4) The student often has a party

a. Der Student macht oft eine Party

el estudiante da a menudo una fiesta

«El estudiante da una fiesta a menudo»

b. *Der Student macht eine Party oft

el estudiante da una fiesta a menudo

«El estudiante da una fiesta a menudo»

c. *Oft der Student hat eine Party

a menudo el estudiante tiene una fiesta

«El estudiante da una fiesta a menudo»

(iii) en la interlengua de algunos aprendices cuya L2 es el alemán (como el Grupo VII que no ha estado expuesto al inglés) aparece el orden incorrecto [ ${ }^{*} \mathrm{~S}$ adv $\left.\mathrm{VO}\right]$ propio del inglés

Por lo que respecta a la segunda pregunta, en base a los datos analizados no parece existir influencia de la L2 a la hora de construir oraciones simples con tiempos compuestos en las que se incluyen adverbios. En la traducción castellano-alemán no se han producido errores de este tipo y en la traducción euskera-alemán tampoco hemos encontrado errores siguiendo el patrón de la construcción inglesa. Del mismo modo, tampoco podemos decir que exista influencia de la L1 puesto que surgen órdenes que no son propios de la L1, como ilustra (5):
(5) *Immer Maria hat Anton geliebt siempre Maria ha Anton amado «Maria siempre ha amado a Anton»

Todo ello parece indicar que los órdenes encontrados surgen como resultado de la opcionalidad de los movimientos que pueden explicarse a partir de una deficiencia en el valor de fuerza de los rasgos (Beck 1998ab; Eubank 1994/96, entre otros).

En cuanto a la tercera pregunta, si los aprendices de alemán como L3 no realizan la inversión del sujeto como en la L2, tenemos que decir que todas las pruebas administradas reflejan la existencia de este error en la interlengua de los aprendices. Sin embargo, en la prueba de juicios de gramaticalidad, en la traducción inglés-alemán y en la traducción euskera-alemán, también se aprecia la utilización del orden SOV tras el elemento adverbial, siendo este orden una secuencia que no se da ni en castellano ni en inglés, aunque sí en euskera. Si únicamente los Grupos III y IV hubiesen presentado el orden SOV, hubiéramos podido concluir que se trata de influencia del euskera. Sin embargo, el orden SOV aparece tanto en los Grupos I y II, como en los grupos de control que nunca han recibido clases de euskera. En (6) mostramos un ejemplo tomado de la prueba de juicios de gramaticalidad:

(6) *Heute Peter seiner Freundin die Ubr gezeigt hat hoy Peter a su novia el reloj mostrado ha «Hoy Peter le ha mostrado a su novia el reloj»

A todas estas respuestas que refutan la hipótesis de influencia de la L2, se unen también dos hechos que hemos constatado a lo largo del análisis de los datos:

(i) Los datos del Grupo VII (para los que el alemán es su L2) reflejan la misma tipología de errores que los grupos cuya L3 es el alemán

(ii) Inexistencia de diferencias significativas entre el Grupo VII y los grupos que tienen como L3 el alemán y existencia de diferencias significativas entre el Grupo VII y los Grupos $\mathrm{V}$ y VI. Todo ello viene a indicar que en estadios anteriores de aprendizaje a los de los Grupos V y VI, aparecen errores semejantes a los de los grupos experimentales, como los que se presentan a continuación:

a) Sobregeneralización de $\mathrm{SOV}$ a construcciones que requieren SVO en alemán nativo, como en (7):

(7) *Das Kind der Mutter das Buch gegeben hat

el niño a la madre el libro dado ha

«El niño le ha dado el libro a la madre»

b) Se acepta *advSVO para las oraciones que precisan la inversión del sujeto, como en (8):

(8) "Heute Peter hat gezeigt seiner Freundin die Uhr hoy Pedro ha mostrado a su novia el reloj «Hoy Pedro le ha mostrado a su novia el reloj» 
c) Sobregeneralización de SOV a construcciones que requieren VSO, como ilustra (9):

(9) *Heute Peter seiner Freundin die Ubr gezeigt hat hoy Pedro a su novia el reloj mostrado ha «Hoy Pedro le ha mostrado a su novia el reloj»

d) Se acepta el orden SVO en la oración subordinada, como en $(10)$ :

(10) *Weil ich habe ein neues Auto, ich bin frob porque yo tengo un nuevo coche, yo estoy feliz «Estoy feliz porque tengo un coche nuevo»

e) Se acepta el orden VSO en la oración subordinada, como se observa en (11):

(11) *Wenn einkaufen geht meine Mutter, braucht sie Geld cuando a comprar va mi madre, necesita ella dinero «Cuando mi madre va a comprar, necesita dinero»

f) Se atribuyen rasgos [LC] a la conjunción coordinante, como se aprecia en (12):

(12) *Ich gehe ins Bett, oder ich das Fussballspiel sehe yo voy a la cama, o yo el partido de fútbol veo «Me voy a la cama o veo el partido de fútbol»

g) Se acepta el orden VSO después de la conjunción coordinante, como en (13):

(13) *Er hat gearbeitet und hat sie geschlafen él ha trabajado y ha ella dormido «Él ha trabajado y ella ha dormido»

En este sentido, nuestra investigación avala los hallazgos de $\mathrm{Ha}$ kannson et al. (2002) puesto que igualmente no confirman la hipótesis de influencia de inglés a nivel sintáctico.

En caso de que no se produjera transferencia, predecíamos otras dos tendencias al comienzo de este artículo que podíamos llegar a observar en nuestros datos: refijación u opcionalidad. En cuanto a la refijación del parámetro de ascenso verbal, nuestros datos no lo confirman puesto que en la interlengua de los aprendices se observa una alta frecuencia de errores que demuestran que el parámetro de ascenso verbal no se ha refijado, como es el caso del uso del orden SVO tras el adverbio o el uso de VSO tras el complementante, entre otros, como ilustran (14) y (15):

(14) *In der Abend ich habe gegessen en la tarde yo he comido «Por la tarde he comido»

(15) *Wenn habe ich fertig lernen cuando he yo acabado aprender «Cuando he terminado de aprender»

Por otro lado, los aprendices no muestran preferencias por una estructura determinada, lo que se traduce en la presencia de movimientos opcionales del verbo y del objeto, como en (16) y (17):

(16) a. Dann habe ich gegessen

luego he yo comido

«Luego he comido»

b. *Dann ich habe gegessen

luego yo he comido

«Luego he comido»

(17) a. *Ich habe trinken Milch mit Cola-Cao

Yo he bebido leche con Cola-Cao

«He bebido leche con Cola-Cao»

b. I habe ins Kino gehen

yo he al cine ir

«Yo he ido al cine»

En (16) observamos que un mismo aprendiz en la prueba de producción libre utiliza unas veces un orden correcto VSO tras el elemento adverbial y en otras ocasiones el orden incorrecto SVO. Del mismo modo, en (17) podemos apreciar cómo un mismo aprendiz no produce en unas ocasiones el movimiento obligatorio del objeto y en otras sí.

En este sentido, nuestro estudio avala los hallazgos de otros autores como Beck (1998ab), Grümpel (2000), Hawkins y Chan (1997) o Klein y Casco (1999), entre otros, puesto que todos ellos han encontrado evidencia de movimientos opcionales en todos los estadios de aprendizaje. Hemos comprobado que los aprendices no tienen preferencia por un orden determinado, lo que parece implicar que el valor de fuerza de los rasgos de concordancia no se encuentra fijado aún.

\section{Conclusiones generales}

A partir del estudio que hemos llevado a cabo sobre la posible influencia del inglés como L2 en la adquisición del orden de palabras del alemán, podemos extraer las siguientes conclusiones:

(i) No se produce transferencia de inglés como L2 al alemán como L3, estando estas dos lenguas emparentadas, en contra de estudios sobre transferencia que se centran en el factor de la distancia lingüística (Adiv 1984; Cenoz 1998, 2001; Corder 1979; Grosera 1998; Möhle 1989; Ringbom 1987; Stedje 1976; Vildomec 1963, entre otros). Asimismo, las percepciones que tienen los estudiantes respecto a la distancia lingüística no parecen coincidir con el grado de proximidad real entre las lenguas implicadas

(ii) Se constata la transferencia a nivel léxico al igual que Ringbom (1983, 1986), Cenoz (2001), Bouvy (2000) y Ecke y Hall (1998)

(iii) Los estadios intermedios parecen caracterizarse por variabilidad e indeterminación, como han sugerido otros autores tales como Papp (2000) o Klein y Casco (1999)

(iv) Los grupos de Modelo A (cuya lengua de instrucción es el castellano) y Modelo D (cuya lengua de instrucción es el 
euskera) presentan la misma tipología de errores que no parecen deberse ni a influencia del castellano ni a influencia del euskera

(v) Los aprendices parecen tener acceso parcial a la GU puesto que el inventario de proyecciones funcionales y de rasgos se encuentra disponible. La dificultad reside en la selección del valor del rasgo o en la interpretación del mismo.

(vi) Los datos apoyan la Hipótesis Inicial de la Sintaxis (HIS) de Platzack y avalan los hallazgos del corpus ZISA, de Hakansson et al. (2002) y Grümpel (2000) puesto que la primera estructura en ser adquirida es el orden SVO

(vii) Implicaciones pedagógicas:
Los datos de las diferentes pruebas confirman que las secuencias VSO y SOV entrañan mayor dificultad en el aprendizaje. Es por ello que en las clases de alemán como lengua extranjera se debería insistir más en estas estructuras. Según el corpus ZISA, la secuencia VSO se aprendería antes que SOV. Sin embargo, nuestro trabajo no confirma este hecho puesto que ambos órdenes resultan difíciles de adquirir si observamos las jerarquías de dificultad obtenidas a partir de cada prueba administrada.

También hemos comprobado la existencia de movimientos opcionales del verbo y del objeto. El tipo de errores que hemos advertido a lo largo de todo el corpus debe hacerse explícito

\section{Referencias}

Adiv, E. (1984), «Language learning strategies: The relation between L1 operating principles and language transfer in L2 development", en R. Andersen, Second Languages: A Cross-linguistic Perspective, Rowley, Newbury House Publishers, pp. 125-142.

Beck, M. L. (1998a), «L2 acquisition and obligatory head movement: English-speaking learners of German and the Local Impairment Hypothesis", Studies in Second Language Acquisition, 20, 311-348.

Bouvy, C. (2000), «Towards the construction of a theory of cross-linguistic transfer", en J. Cenoz y U. Jessner, English in Europe: The Acquisition of a Third Language, Clevedon, Multilingual Matters, pp. 143-156.

Cenoz, J. (1998), "Multilingual education in the Basque Country", en J. Cenoz y F. Genesee, Beyond Bilingualism, Clevedon, Multilingual Matters, pp. 175-191.

(2001), «The effect of linguistic distance, L2 status and age on crosslinguiztic influence in third language» Jessner, Cross-linguistic Influence in Third Language Acquisition. PsycholinguisticPerspectives, Clevedon, Multilingual Matters, pp. 8-20.

Chomsky, N. (1994), "A Minimalist Program for linguistic theory", en K. Hale y S. J. Keyser, The View from Building 20 Essays in Linguistics in Honor of Sylvain Bromberger, Cambridge, MIT Press, pp. 1-52.

(1995), The Minimalist Program, Cambridge, MIT Press.

Clahsen, H. (1988), «Parametrized grammatical theory and language acquisition: A study of the acquisition of verb placement and inflection by children and adults", en S. Flynn y W. O'Neil, Linguistic Theory in Second Language Acquisition, Dordrecht, Kluwer.

Clahsen, H., J. Meisel y M. Pienemann. 1983.
Deutsch als Zweitsprache. Der Spracherwerb ausländischer Arbeiter. Tübingen: Narr.

Corder, S. P. (1979), «Language distance and the magnitude of the learning task", Studies in Second Language Acquisition, 2(1), 27-36.

Ecke, P. y C. Hall. (1998), «Tres niveles de representación mental», Estudios de Lingüística Aplicada, 28, 15-26.

Eubank, L. (1993/94), "On the transfer of parametric values in L2 development", Language Acquisition, 3, 183-208.

Faerch, C. y G. Kaesper. (1986), «Cognitive dimensions of language transfer», en E. Kellerman y M. Sharwood-Smith, Crosslinguistic Influence in Second Language Acquisition, Oxford, Pergamon Press, pp. 49-65.

Fathman, A. (1989), «Word order contrasts and production in three target languages», en W. Dechert y M. Raupach, Interlingual Processes, Tübingen, Gunter Narr, pp. 159-170.

Fries, C. (1945), Teaching and Learning English as a Foreign Language, Ann Arbor, University of Michigan Press.

García Mayo, M. P. (2003), «Age, length of exposure and grammaticality judgements in the acquisition of English as a foreign language», en M. P. García Mayo y M. L. García Lecumberri, Age and the Acquisition of English as a Foreign Language, Clevedon, Multilingual Matters, pp. 94-114.

Gibson, M., B. Hufeisen y G. Libben. (2001), "Learners of German L3 and their production of German prepositional verbs", en J. Cenoz, B. Hufeisen y U. Jessner, pp. 138-148.

Grosera, M. (1998), «Dient das L2-System als ein Fremdsprachenlernmodell?», en B. Hufeisen y B. Lindemann, Tertiärsprachen: Theorien, Modelle, Methoden, Tübingen, Stuffenburg Verlag, pp. 133-144.

Hakansson, G., M.Pienemann y S. Sayehli. (2002),
«Transfer and typological proximity in the context of second language processing", $\mathrm{Se}$ cond Language Research, 18 (3), 250-273.

Hawkins, R. y C. Chan. (1997), "The partial ability of UG in SLA: The failed functional feature hypothesis", Second Language Research, 13 (3), 187-226.

Kellerman, E. (1977), «Towards a characterisation of the strategy of transfer in second language learning», Interlanguage Studies Bulletin, 2 (1), 58-146.

(1986), «An eye for an eye: Crosslinguistic constraints on the development of the L2 lexicon», en E. Kellerman y M. SharwoodSmith, 35-48.

(1987), Aspects of transferability in second language acquisition, Tesis doctoral, Universidad de Nijmegen.

Kohn, K. (1986), "The analysis of transfer", en E. Kellerman y M. Sharwood-Smith, pp. 21-34.

Lado, R. (1957), Linguistics across Cultures, Ann Arbor, University of Michigan Press.

Lardiere, D. (2000), "Mapping features to forms in SLA", en J. Archibald, Second Language Acquisition and Linguistic Theory, Oxford, Blackwell, pp. 102-129.

Martínez Adrián, M. (2001), «El efecto gramatical de inglés como L2 en el aprendizaje de alemán como L3», en A. Moreno y V. Colwell, Recent Perspectivas on Discourse, León, Universidad de León.

(2002), «Una reflexión sobre la posible influencia del inglés como L2 en el aprendizaje del alemán como L3", Interlingüística, 13 (3), 13-20.

(2003), «Una reflexión sobre la posible influencia del inglés como L2 en la adquisición de los auxiliares de perfecto del alemán», Fifty Years of English Studies in Spain(1952-2002). 
en las clases, como sugiere la pedagogía comunicativa con atención a aspectos formales (communicative-focus-on-form, Doughty y Williams 1998). Los alumnos necesitan evidencia negativa en forma de explicaciones gramaticales o en forma de RETROALIMENT correctora (Spada 1997).

Por otro lado, no se confirma la transferencia de L2 a L3 en el nivel sintáctico pero sí en el plano léxico. Hemos constatado la presencia de influencia tanto de las L1s como de la L2 a este nivel y por tanto, convendría hacer referencia en las clases a los posibles errores causados por la influencia de otras lenguas o hacer actividades destinadas a minimizar dicha influencia.
Finalmente, autoras como Papp (2000) y Sorace $(2000,2003)$ consideran que la escasez de input está relacionada con la emergencia de la opcionalidad. Por ello, aumentar las horas de exposición sería determinante. De hecho, en nuestra investigación hemos podido comprobar que los Grupos V y VI que han tenido una exposición más intensa al alemán que el resto de grupos presentan un índice de opcionalidad menor. Tal vez, se debería pensar en una mayor intensidad de la exposición y en el uso de la lengua extranjera como vehículo para la instrucción de otras materias como algunos de los factores que podrían mejorar el nivel de competencia en la lengua extrajera (Pica 2002)
A Commemorative Volume, Santiago de Compostela, Universidad de Santiago de Compostela, pp. 389-397.

(2004/2005), «La hipótesis de la transferencia de la L2 y la adquisición del orden de palabras", Revista Española de Lingüística Aplicada, 17/18, 187-208.

(2005), El papel de la segunda lengua en la adquisición del orden de palabras del alemán como tercera lengua, Tesis doctoral, Universidad del País Vasco/Euskal Herriko Unibertsitatea, Vitoria-Gasteiz.

(2006), «Syntactic optionality in L2 grammars», Interlingüística, [en prensa].

Meisel, J. (1983), «Transfer as a second language strategy", Language and Communication 3 (1), 11-46.

Meisel, J. (1997), «The acquisition of the syntax of negation in French and German: Contrasting first and second language development", Second Language Research, 13, 227-263.

Möhle, D. (1989), «Multilingual interaction in foreign language production", en H. W. Derchert y M. Raupach, pp. 179-194.

Neuner, G. (1996), «Deutsch als zweite Fremdsprache nach Englisch. Überlegungen zur Didaktik und Methodik und zur Lehrmaterialentwicklung für die 'Drittsprache Deutsch'», Deutsch als Fremdsprache, 4, 211-217.

Pica, T. (2002), «Subject-matter content: How does it assist the interactional and linguistic needs of classroom language learning? ", The Modern Language Journal, 86 (1), 1-19.

Platzack, C. (1996), «The initial hypothesis of syntax: A Minimalist perspective on language acquisition and attrition", en $\mathrm{H}$. Clahsen, Generative Perspectives on Language Acquisition, Amsterdam, John Benjamins, 396-414.
Prévost, P. y L. White. (2000), «Missing surface inflection or impairment in Second Language Acquisition? Evidence from tense and agreement", Second Language Research, 16, 2, 103-133.

Ringbom, H. (1983), «Borrowing and lexical transfer», Applied Linguistics, 4 (3), 207-12.

(1987), The Role of the First Language in Foreign Language Learning, Clevedon, Multilingual Matters.

Robertson, D. y A. Sorace. (1999), «Losing the V2 constraint», en E. C. Klein y G. Martohardjono, The Development of Second Language Grammars, Amsterdam, John Benjamins, 317-367.

Schwartz, B. D. y R. Sprouse. (1994), «Word order and nominative case in nonnative language acquisition: A longitudinal study of (L1 Turkish) German Interlanguage», en T. Hoekstra y B. F. Schwartz, Language Acquisition Studies in Generative Grammar, pp. 317-368.

(1996), «L2 cognitive states and the Full Transfer/Full Access Model», Second Language Research, 12, 40-72.

Sorace, A. (2000), «Syntactic optionality in nonnative grammars", Second Language Research, 16 (2), 93-102.

(2003), «Near-nativeness», en C. J. Doughty y M. L. Long, The Handbook of Second Language Acquisition, Oxford, Blackwell, pp. 130-151.

Spada, N. (1997), «Form-Focussed instruction and second language acquisition: A review of classroom and laboratory research", Language Teaching, 30, 73-87.

Stedje, A. (1976), «Interferenz von Muttersprache und Zweitsprache auf eine dritte Sprache beim freien Sprechen-ein Vergleich", Zielsprache Deutsch, 1, 15-21.
Tsimpli, I. (1997), «Resumptive strategies and L2 acquisition: A minimalist account», en BUCLD 21 Proceedings, Somerville, Cascadilla Press, pp. 639-655.

Vainikka, A. y M. Young-Scholten. (1994), «Direct access to X-bar theory: Evidence from Korean and Turkish adults learning German», en T. Hoekstra y B. D. Schwartz, pp. 265-316.

(1996), "Gradual development of L2 phrase structure», Second Language Research, 12, 7-39.

Vildomec, V. (1963), Multilingualism, Leyden, Syhoff.

Vogel, T. (1992), «Englisch und Deutsch gibt es immer Krieg», Zielsprache Deutsch, 23 (2), 95-99.

Welge, P. K. (1987), «Deutsch nach Englisch. Deutsch als dritte Sprache», en S. Ehlers y G.L. Kardener, Regionale Aspekte des Grundstudiums Germanistik, München, Iudicium, pp. 189-225.

White, L. (1987), «Markedness and second language acquisition. The question of transfer.", Studies in Second Language Acquisition, 9, 261-285.

Zwart, J. W. (1997a), Dutch Syntax: A Minimalist Approach,. Dordrecht, Kluwer.

(1997b), «The Germanic SOV languages and the Universal Base Hypothesis", en L. Haegeman, The New Comparative Syntax, Harlow, Longman, pp. 246-267.

\section{Nota}

1 Los resultados que se presentan a continuación forman parte de mi tesis doctoral titulada «El papel de la L2 en la adquisición del orden de palabras del alemán como L3» 